\title{
REMARKS ON NON-LOCAL INVARIANTS OF MARTINET'S SINGULAR SYMPLECTIC STRUCTURES
}

\author{
WOJCIECH DOMITRZ \\ Warsaw University of Technology, Faculty of Mathematics and Information Science \\ Plac Politechniki 1, 00-661 Warszawa, Poland \\ E-mail: domitrz@mini.pw.edu.pl
}

1. Introduction. The fundamental result for symplectic topology is Gromov's nonsqueezing theorem.

Theorem 1 (Gromov's Nonsqueezing Theorem). Let

$$
\omega_{0}=\sum_{i=1}^{n} d p_{i} \wedge d q_{i}
$$

be the standard symplectic structure on $\mathbb{R}^{2 n}$. If there is a symplectic embedding

$$
B^{2 n}(r) \hookrightarrow Z^{2 n}(R),
$$

where $B^{2 n}(r)=\left\{(p, q) \in \mathbb{R}^{2 n}:|p|^{2}+|q|^{2} \leq r^{2}\right\}$ is a standard ball and

$$
Z^{2 n}(R)=B^{2}(R) \times \mathbb{R}^{2 n-2}=\left\{(p, q) \in \mathbb{R}^{2 n}: p_{1}^{2}+q_{1}^{2} \leq R^{2}\right\}
$$

is a symplectic cylinder, then

$$
r \leq R
$$

Gromov proves this theorem using $J$-holomorphic curves ([9]). There are other proofs of this theorem: a proof due to Viterbo which uses generating functions ([20]) and a proof due to Hofer and Zehnder which is based on the calculus of variations ([10]).

This theorem was extended to arbitrary symplectic manifold $(M, \omega)$ by Lalonde and $\operatorname{McDuff}([12])$.

THEOREM 2. If $(M, \omega)$ is any symplectic manifold of dimension $2 n$, there is a symplectic embedding of the standard ball $B^{2 n+2}(r)$ into the cylinder $\left(B^{2}(R) \times M, d p \wedge d q \oplus \omega\right)$ only if $r \leq R$.

2000 Mathematics Subject Classification: 57R17.

Research of the author partially supported by Special Program "Dynamics of Complex Systems", Warsaw University of Technology.

The paper is in final form and no version of it will be published elsewhere. 
Gromov's nonsqueezing theorem is crucial for the proof of rigidity of symplectomorphisms. It is also the most basic geometric expression of this rigidity (see [14], [10]). This theorem makes possible to define a new symplectic invariant (a symplectic capacity) Gromov width.

Another problem which visualize symplectic invariants is the symplectic camel problem. Let

$$
W=\left\{(p, q) \in \mathbb{R}^{2 n}: p_{1}=0\right\}
$$

and

$$
H_{r}=\left\{(p, q) \in \mathbb{R}^{2 n}:|p|^{2}+|q|^{2}<r^{2}\right\} .
$$

We ask if there exists a continuous family (an isotopy) of symplectic embeddings $[0,1] \ni$ $t \mapsto \Phi_{t}: B^{2 n}(R) \rightarrow \mathbb{R}^{2 n}$, such that $\Phi_{t}\left(B^{2 n}(R)\right) \subset \mathbb{R}^{2 n} \backslash\left(W \backslash H_{r}\right)$ for every $t \in[0,1]$ and $\Phi_{0}\left(B^{2 n}(R)\right), \Phi_{1}\left(B^{2 n}(R)\right)$ are in different components of $\mathbb{R}^{2 n} \backslash W$. The question was asked by Arnold. McDuff and Traynor in [15] and Viterbo in [20] prove that such symplectic isotopy exists if and only if $R<r$. McDuff and Traynor use Gromov's methods developed to prove the nonsqueezing theorem and Viterbo's proof uses generating functions.

In this paper we consider similar problems for Martinet's singular symplectic form $\omega=x d x \wedge d y+\sum_{i=1}^{n-1} d p_{i} \wedge d q_{i}$ on $\mathbb{R}^{2 n}$. This closed 2-form is also called a folded symplectic form (see [2]). It is considered in [13], [17], [11], [4], [5], [3] and [2].

Now we recall some basic facts on the local classification of singularities of differential closed 2-forms on $\mathbb{R}^{2 n}$ for $n \geq 2$ ([13]).

Let $\alpha$ be a germ of a closed 2 -form on $\mathbb{R}^{2 n}$ at 0 . We define

$$
\Sigma_{k}(\alpha)=\left\{z \in \mathbb{R}^{2 n}:\left.\operatorname{rank} \alpha\right|_{z}=2 n-k\right\}, k \text { is even. }
$$

Let $\alpha^{n}=f \Omega$, where $\Omega$ is the volume form on $\mathbb{R}^{2 n}$.

(i) If $f(0) \neq 0$ then $\alpha$ is a germ of a symplectic form (denoted by $\Sigma_{0}$ ) and by Darboux theorem we obtain

$$
\alpha=\sum_{i=1}^{n} d x_{i} \wedge d y_{i}
$$

in local coordinates around $0 \in \mathbb{R}^{2 n}$.

(ii) Next we assume $f(0)=0$ while $(d f)(0) \neq 0$. We have $\Sigma_{2}(\alpha)=\{f=0\}$. If $\left(\left.\alpha\right|_{\Sigma_{2}(\alpha)}\right)^{n-1}(0) \neq 0$ then in local coordinates around $0 \in \mathbb{R}^{2 n}$

$$
\alpha=x_{1} d x_{1} \wedge d y_{1}+\sum_{i=2}^{n} d x_{i} \wedge d y_{i}
$$

and this type of singularity is denoted by $\Sigma_{2,0}$ (and called Martinet's singular symplectic form).

Both types of forms $\Sigma_{0}, \Sigma_{2,0}$ are locally stable (see [13]).

Let $\omega=x d x \wedge d y+\sum_{i=1}^{n-1} d p_{i} \wedge d q_{i}$ denote Martinet's singular symplectic structure on $\mathbb{R}^{2 n}$. Then

$$
\Sigma=\Sigma_{2}(\omega)=\left\{z \in \mathbb{R}^{2 n}:\left.\omega^{n}\right|_{z}=0\right\}=\left\{z \in \mathbb{R}^{2 n}: x=0\right\}
$$

is a hypersurface of degeneration of $\omega$. 
2. Nonsqueezing for Martinet's singular symplectic structure on $\mathbb{R}^{2 n}$. Let

$$
B^{2 n}(r)=\left\{z=(x, y, p, q) \in \mathbb{R}^{2 n}:(x, y) \in \mathbb{R}^{2},|z| \leq r\right\}
$$

be the ball of radius $r$ in $\mathbb{R}^{2 n}$ and

$$
Z^{2 n}(R)=\left\{z=(x, y, p, q) \in \mathbb{R}^{2 n}: p_{1}^{2}+q_{1}^{2} \leq R^{2}\right\}
$$

be the cylinder in $\mathbb{R}^{2 n}$. Then it is easy to prove that

Proposition 1. If there is an embedding $\Phi: B^{2 n}(r) \hookrightarrow Z^{2 n}(R)$ preserving $\omega$ then $r \leq R$.

Proof. It is obvious that $\Phi$ must preserve the hypersurface

$$
\Sigma=\left\{z \in \mathbb{R}^{2 n}: x=0\right\}
$$

because $\Phi$ preserves $\omega$. Let us consider $\phi=\left.\Phi\right|_{\Sigma}$. Let $B^{2 n-1}(r)=B^{2 n}(r) \cap \Sigma, Z^{2 n-1}(R)=$ $Z^{2 n}(R) \cap \Sigma$ and $\omega_{1}=\left.\omega\right|_{\Sigma}=\sum_{i=1}^{n-1} d p_{i} \wedge d q_{i}$. The kernel of $\omega_{1}$ is spanned by $\partial / \partial y$. It is tangent to the boundary of $Z^{2 n-1}(R)$ and it is tangent to the boundary of $B^{2 n-1}(r)$ on the set

$$
S^{2 n-3}(r)=\left\{(y, p, q) \in \Sigma: y=0,|p|^{2}+|q|^{2}=r^{2}\right\} .
$$

Let us consider $B^{2 n-2}(r)=B^{2 n-1}(r) \cap\{(y, p, q) \in \Sigma: y=0\}$. Its boundary is $S^{2 n-3}(r)$ and the kernel of $\omega_{1}$ is transversal to it. Let us consider $\psi=\left.\pi_{y} \circ \phi\right|_{B^{2 n-2}(r)}$ where $\pi_{y}$ is the projection of $Z^{2 n-1}(R)$ onto $Z^{2 n-2}(R)=Z^{2 n-1}(R) \cap\{(y, p, q) \in \Sigma: y=0\}$ along $y$-axis. It is an embedding, because $\partial / \partial y$ is transversal to $\phi\left(B^{2 n-2}(r)\right)$. $\psi$ preserves the symplectic form $\sum_{i=1}^{n-1} d p_{i} \wedge d q_{i}$ on $\mathbb{R}^{2 n-2}$ and maps $B^{2 n-2}(r)$ - the standard ball of radius $r$ into $Z^{2 n-2}(R)$ - the standard symplectic cylinder of radius $R$. Therefore $r \leq R$ by Gromov's nonsqueezing theorem.

Proposition 1 is true for every cylinder $Z$, such that the kernel of $\left.\omega\right|_{\Sigma}$ is tangent to $\partial Z \cap \Sigma$. But this is not a typical position. The kernel of $\left.\omega\right|_{\Sigma}$ is transversal to $\partial Z \cap \Sigma$ for a typical position of a cylinder $Z$. It is an open problem if the nonsqueezing theorem is true for a typical position of a cylinder $Z$. The method of restriction to $\Sigma$ does not work in this case. This is a consequence of the following

Proposition 2. If $\omega_{1}=\sum_{i=1}^{n-1} d p_{i} \wedge d q_{i}$ is a closed 2-form on $\mathbb{R}^{2 n-1}$ then for any $R, r>0$ there exists an embedding preserving $\omega_{1}$ of

$$
B^{2 n-1}(r)=\left\{z=(y, p, q) \in \mathbb{R}^{2 n-1}:|z| \leq r\right\}
$$

into

$$
Z^{2 n-1}(R)=\left\{z=(y, p, q) \in \mathbb{R}^{2 n-1}: y^{2}+q_{1}^{2} \leq R^{2}\right\}
$$

Proof. It is easy to check that

$$
\Phi(y, p, q)=\left(\frac{R y}{r}, \frac{r p_{1}}{R}, p_{2}, \ldots, p_{n-1}, \frac{R q_{1}}{r}, q_{2}, \ldots, q_{n-1}\right)
$$

satisfies these conditions. 
3. The camel problem for Martinet's singular symplectic structure on $\mathbb{R}^{2 n}$. Let $W$ be a hyperplane in $\mathbb{R}^{2 n}$, transversal to $\Sigma$, and $0 \in W$. Let $H_{r}=\left\{z \in \mathbb{R}^{2 n}:|z|<r\right\}$ ( $W$ is a "wall" and $H_{r}$ is a "hole" of a radius $r$ in the wall). We ask if there exists a continuous family (an isotopy) of embeddings $[0,1] \ni t \mapsto \Phi_{t}: B^{2 n}(R) \rightarrow \mathbb{R}^{2 n}$, such that $\Phi_{t}\left(B^{2 n}(R)\right) \subset \mathbb{R}^{2 n} \backslash\left(W \backslash H_{r}\right), \Phi_{t}^{*} \omega=\omega$ for every $t \in[0,1]$ and $\Phi_{0}\left(B^{2 n}(R)\right)$ and $\Phi_{1}\left(B^{2 n}(R)\right)$ are in different components of $\mathbb{R}^{2 n} \backslash W$. This is an analog of the camel problem for the Martinet singular symplectic structure.

Firstly we find a normal form for the hyperplane $W$.

In a typical position $W$ is transversal to the kernel of $\left.\omega\right|_{\Sigma}$ on $W \cap \Sigma$. The kernel of $\left.\omega\right|_{\Sigma}$ is spanned by $\partial / \partial y$. If

$$
W=\left\{z \in \mathbb{R}^{2 n}: A x+B y+\sum_{i=1}^{n-1} C_{i} p_{i}+D_{i} q_{i}=0\right\}
$$

then $B \neq 0$. Therefore by a diffeomorphism of the form $\Psi(z)=\left(x, y+\frac{A}{B} x, p, q\right)$, which preserves $\omega$, we reduce $W$ to $\left\{z \in \mathbb{R}^{2 n}: y+\sum_{i=1}^{n-1} E_{i} p_{i}+F_{i} q_{i}=0\right\}$. If $E_{k}^{2}+F_{k}^{2} \neq 0$ we may assume that $E_{k} \neq 0$ (otherwise we may use a diffeomorphism

$$
\left.\Phi(z)=\left(x, y, p_{1}, \ldots, p_{k-1}, q_{k}, p_{k+1}, \ldots, p_{n}, q_{1}, \ldots, q_{k-1},-p_{k}, q_{k+1}, \ldots, q_{n}\right)\right) .
$$

Now we transform $W$ to $\left\{z \in \mathbb{R}^{2 n}: y+p_{k}+\sum_{i=1, i \neq k}^{n-1} E_{i} p_{i}+F_{i} q_{i}=0\right\}$ by a diffeomorphism

$$
\Theta(z)=\left(x, y, p_{1}, \ldots, p_{k-1}, E_{k} p_{k}+F_{k} q_{k}, p_{k+1}, \ldots, p_{n}, q_{1}, \ldots, q_{k-1}, \frac{q_{k}}{E_{k}}, q_{k+1}, \ldots, q_{n}\right),
$$

which preserves $\omega$. Finally by a diffeomorphism

$$
\Gamma(z)=\left(x, y+p_{k}, p_{1}, \ldots, p_{k-1}, p_{k}, p_{k+1}, \ldots, p_{n}, q_{1}, \ldots, q_{k-1}, q_{k}+\frac{x_{1}^{2}}{2}, q_{k+1}, \ldots, q_{n}\right),
$$

which preserves $\omega$, we reduce $W$ to $\left\{z \in \mathbb{R}^{2 n}: y+\sum_{i=1, i \neq k}^{n-1} E_{i} p_{i}+F_{i} q_{i}=0\right\}$. If we repeat these transformations for each $k$ such that $E_{k}^{2}+F_{k}^{2} \neq 0$ then we reduce $W$ to $\left\{z \in \mathbb{R}^{2 n}: y=0\right\}$.

If $W$ is not transversal to the kernel of $\left.\omega\right|_{\Sigma}$ and is transversal to $\Sigma$ then it has the form $W=\left\{z \in \mathbb{R}^{2 n}: A x+\sum_{i=1}^{n-1} C_{i} p_{i}+D_{i} q_{i}=0\right\}$ where $\sum_{i=1}^{n-1} C_{i}^{2}+D_{i}^{2} \neq 0$. We may assume that $C_{k} \neq 0$ for some $k$ (otherwise $D_{k} \neq 0$ for some $k$ and we may use a diffeomorphism

$$
\left.\Phi(z)=\left(x, y, p_{1}, \ldots, p_{k-1}, q_{k}, p_{k+1}, \ldots, p_{n}, q_{1}, \ldots, q_{k-1},-p_{k}, q_{k+1}, \ldots, q_{n}\right)\right) .
$$

Now we transform $W$ to $\left\{z \in \mathbb{R}^{2 n}: A x+p_{k}+\sum_{i=1, i \neq k}^{n-1} C_{i} p_{i}+D_{i} q_{i}=0\right\}$ by a diffeomorphism

$$
\Theta(z)=\left(x, y, p_{1}, \ldots, p_{k-1}, C_{k} p_{k}+D_{k} q_{k}, p_{k+1}, \ldots, p_{n}, q_{1}, \ldots, q_{k-1}, \frac{q_{k}}{C_{k}}, q_{k+1}, \ldots, q_{n}\right),
$$

which preserves $\omega$. If $\sum_{i=1, i \neq k}^{n-1} C_{i}^{2}+D_{i}^{2} \neq 0$ then in the same way we may reduce $W$ to $\left\{z \in \mathbb{R}^{2 n}: A x+p_{k}+p_{l}+\sum_{i=1, i \neq k, l}^{n-1} C_{i} p_{i}+D_{i} q_{i}=0\right\}$ for some $l \neq k$. By a diffeomorphism

$$
\Delta(z)=\left(x, y, p_{1}, \ldots, p_{k-1}, p_{k}+p_{l}, p_{k+1}, \ldots, p_{n}, q_{1}, \ldots, q_{l-1}, q_{l}-q_{k}, q_{l+1}, \ldots, q_{n}\right)
$$


we reduce $W$ to $\left\{z \in \mathbb{R}^{2 n}: A x+p_{k}+\sum_{i=1, i \neq k, l}^{n-1} C_{i} p_{i}+D_{i} q_{i}=0\right\}$. Repeating these transformations for each $l$ such that $C_{l}^{2}+D_{l}^{2} \neq 0$ we reduce $W$ to $\left\{z \in \mathbb{R}^{2 n}: A x+p_{k}=0\right\}$. If $A \neq 0$ then we may reduce $W$ to $\left\{z \in \mathbb{R}^{2 n}: x+p_{1}=0\right\}$ and if $A=0$ then we may reduce $W$ to $\left\{z \in \mathbb{R}^{2 n}: p_{1}=0\right\}$ by diffeomorphisms which preserve $\omega$. Thus we obtain

Proposition 3. If a hyperplane $W$ is transversal to $\Sigma$ then there exists a diffeomorphism $\Phi:\left(\mathbb{R}^{2 n}, 0\right) \rightarrow\left(\mathbb{R}^{2 n}, 0\right)$ such that $\Phi^{\star} \omega=\omega$ and

$$
\Phi^{-1}(W)=\left\{z \in \mathbb{R}^{2 n}: y=0\right\}
$$

(if the kernel of $\left.\omega\right|_{\Sigma}$ is transversal to $W$ ) or

$$
\Phi^{-1}(W)=\left\{z \in \mathbb{R}^{2 n}: x+p_{1}=0\right\}
$$

(if the kernel of $\left.\omega\right|_{\Sigma}$ is tangent to $W$ and the rank at $\left.\omega\right|_{W}$ at 0 is maximal) or

$$
\Phi^{-1}(W)=\left\{z \in \mathbb{R}^{2 n}: p_{1}=0\right\}
$$

(if the kernel of $\left.\omega\right|_{\Sigma}$ is tangent to $W$ and the rank at $\left.\omega\right|_{W}$ at 0 is not maximal).

Now it is easy to prove

Proposition 4. If a hyperplane $W$ is transversal to $\Sigma$ and the kernel of $\left.\omega\right|_{\Sigma}$ is tangent to $W$ then there exists an isotopy of embeddings $[0,1] \ni t \mapsto \Phi_{t}: B^{2 n}(R) \rightarrow \mathbb{R}^{2 n}$, such that $\Phi_{t}\left(B^{2 n}(R)\right) \subset \mathbb{R}^{2 n} \backslash\left(W \backslash H_{r}\right), \Phi_{t}^{*} \omega=\omega$ for every $t \in[0,1]$, and $\Phi_{0}\left(B^{2 n}(R)\right)$ and $\Phi_{1}\left(B^{2 n}(R)\right)$ are in different components of $\mathbb{R}^{2 n} \backslash W$ if and only if $R<r$, where $r$ is a radius of the hole $H_{r}$.

Proof. By Proposition 3 we may assume that $W$ is

$$
\left\{z \in \mathbb{R}^{2 n}: x+p_{1}=0\right\}
$$

or

$$
\left\{z \in \mathbb{R}^{2 n}: p_{1}=0\right\} .
$$

Let us assume that there exists an isotopy $\Phi_{t}$ which satisfies these conditions and let us consider $\phi_{t}=\left.\Phi_{t}\right|_{\Sigma \cap B^{2 n}(R)}: B^{2 n-1}(R) \rightarrow \mathbb{R}^{2 n-1}$ for $t \in[0,1]$. In both cases $W \cap \Sigma$ is $\left\{z \in \mathbb{R}^{2 n}: p_{1}=0\right\}$. Now we use the same argument as in the proof of Proposition 1. Let $B^{2 n-1}(R)=B^{2 n}(R) \cap \Sigma$ and $\omega_{1}=\left.\omega\right|_{\Sigma}=\sum_{i=1}^{n-1} d p_{i} \wedge d q_{i}$. The kernel of $\omega_{1}$ is spanned by $\partial / \partial y$. It is tangent to the boundary of $B^{2 n-1}(R)$ on a set

$$
S^{2 n-3}(R)=\left\{(y, p, q) \in \Sigma: y=0,|p|^{2}+|q|^{2}=R^{2}\right\} .
$$

Let us consider the submanifold $B^{2 n-2}(R)=B^{2 n-1}(R) \cap\{(y, p, q) \in \Sigma: y=0\}$. Its boundary is $S^{2 n-3}(R)$ and the kernel of $\omega_{1}$ is transversal to this submanifold. Let us consider $\psi_{t}=\left.\pi_{y} \circ \phi_{t}\right|_{B^{2 n-2}(R)}$ where $\pi_{y}$ is a projection of $\mathbb{R}^{2 n-1}$ onto $\mathbb{R}^{2 n-2}=$ $\{(y, p, q) \in \Sigma: y=0\}$ along $y$-axis. It is an embedding, because $\partial / \partial y$ is transversal to $\phi_{t}\left(B^{2 n-2}(R)\right) . \psi_{t}$ preserves the symplectic form $\sum_{i=1}^{n-1} d p_{i} \wedge d q_{i}$ on $\mathbb{R}^{2 n-2} \cdot \pi_{y}(W \cap \Sigma)=$ $\left\{(p, q) \in \mathbb{R}^{2 n-2}: p_{1}=0\right\}$ and $\pi_{y}\left(H_{r} \cap \Sigma\right)=\left\{(p, q) \in \mathbb{R}^{2 n-2}:|p|^{2}+|q|^{2}<r^{2}\right\}$. Therefore if $\psi_{t}$ exists then $R<r$ by the symplectic camel theorem.

If the kernel of $\left.\omega\right|_{\Sigma}$ is transversal to $W$ then we cannot use the same method to prove the camel theorem. But one can prove the following. 
Proposition 5. If a hyperplane $W$ is transversal to the kernel of $\left.\omega\right|_{\Sigma}, R<2$ and

$$
r<\frac{R^{2}}{4}
$$

then there is no isotopy of embeddings $[0,1] \ni t \mapsto \Phi_{t}: B^{2 n}(R) \rightarrow \mathbb{R}^{2 n}$, such that $\Phi_{t}\left(B^{2 n}(R)\right) \subset \mathbb{R}^{2 n} \backslash\left(W \backslash H_{r}\right), \Phi_{t}^{*} \omega=\omega$ for every $t \in[0,1]$, and $\Phi_{0}\left(B^{2 n}(R)\right)$ and $\Phi_{1}\left(B^{2 n}(R)\right)$ are in different components of $\mathbb{R}^{2 n} \backslash W$, where $r$ is a radius of the hole $H_{r}$.

Proof. By Proposition 3 we may assume that $W$ is $\left\{z \in \mathbb{R}^{2 n}: y=0\right\}$. Let us assume that there exists an isotopy $\Phi_{t}$, which satisfies these conditions. Let

$$
M^{+}=\left\{z \in \mathbb{R}^{2 n}: x>0\right\}, M^{-}=\left\{z \in \mathbb{R}^{2 n}: x<0\right\} .
$$

It is easy to see that $\Phi_{t}\left(B^{2 n}(R) \cap M^{+}\right) \subset M^{+}$or $\Phi_{t}\left(B^{2 n}(R) \cap M^{+}\right) \subset M^{-}$. We assume that $\Phi_{t}\left(B^{2 n}(R) \cap M^{+}\right) \subset M^{+}$. Let

$$
\Theta: M^{+} \ni(x, y, p, q) \mapsto(\sqrt{2 x}, y, p, q) \in M^{+} .
$$

It is easy to see that $\Theta^{\star} \omega=\omega_{0}=d x \wedge d y+\sum_{i=1}^{n-1} d p_{i} \wedge d q_{i}$,

$$
P(R)=\Theta^{-1}\left(B^{2 n}(R) \cap M^{+}\right)=\left\{(x, y, p, q) \in \mathbb{R}^{2 n}: 2 x+y^{2}+|p|^{2}+|q|^{2}<R^{2}, x>0\right\}
$$

and

$$
P(r)=\Theta^{-1}\left(H_{r} \cap M^{+}\right)=\left\{(x, y, p, q) \in \mathbb{R}^{2 n}: 2 x+y^{2}+|p|^{2}+|q|^{2}<r^{2}, x>0\right\} .
$$

It is obvious that the ball $B^{2 n}\left(R^{2} / 4\right)$ is symplectically embedded in $P(R)$, because $R<2$. Let $\Psi$ denote such an embedding. On the other hand $P(r)$ is symplectically embedded in the ball $B^{2 n}(r)$. Thus the mapping

$$
\Theta^{-1} \circ \Phi_{t} \circ \Theta \circ \Psi: B^{2 n}\left(R^{2} / 4\right) \rightarrow \mathbb{R}^{2 n}
$$

defines an isotopy of symplectic embeddings such that $\Phi_{t}\left(B^{2 n}\left(R^{2} / 4\right)\right) \subset \mathbb{R}^{2 n} \backslash\left(W \backslash H_{r}\right)$ for every $t \in[0,1]$, and $\Phi_{0}\left(B^{2 n}\left(R^{2} / 4\right)\right), \Phi_{1}\left(B^{2 n}\left(R^{2} / 4\right)\right)$ are in different components of $\mathbb{R}^{2 n} \backslash W$. By the symplectic camel theorem we get that such isotopy does not exist if $r<R^{2} / 4$.

It is an open problem if the camel theorem for Martinet's singular symplectic structures is true for $R^{2} / 4 \leq r<R$.

Acknowledgments. I wish to express my thanks to S. Janeczko for many helpful conversations and remarks. I am also grateful to the referee for many useful suggestions.

\section{References}

[1] V. I. Arnol'd, First steps of symplectic topology, Uspekhi Mat. Nauk 41:6 (1986), 3-18 (in Russian); English transl.: Russian Math. Surveys 41:6 (1986), 1-21.

[2] A. Cannas da Silva, V. Guillemin, C. Woodward, On the unfolding of folded symplectic structures, Math. Res. Lett. 7 (2000), 35-53.

[3] W. Domitrz, Singularities of differential 1-forms on a manifold with boundary, in: Quantization, Coherent States, and Poisson Structures (Białowieża, 1995), PWN, Warsaw, 1998, $227-232$. 
[4] W. Domitrz, S. Janeczko, Normal forms of symplectic structures on the stratified spaces, Colloq. Math. 68 (1995), 101-119.

[5] W. Domitrz, S. Janeczko, On Martinet's singular symplectic structures, in: Singularities and Differential Equations, Banach Center Publ. 33, Warsaw, 1996, 51-59.

[6] M. Golubitsky, V. Guillemin, Stable Mappings and their Singularities, Graduate Texts in Math. 14, Springer, New York, 1973.

[7] M. Golubitsky, D. Tischler, A survey on the singularities and stability of differential forms, Astérisque 59-60 (1978), 43-82.

[8] M. Golubitsky, D. Tischler, An example of moduli for singular symplectic forms, Invent. Math. 38 (1977), 219-225.

[9] M. Gromov, Pseudo-holomorphic curves in symplectic manifolds, Invent. Math. 82 (1985), 307-347.

[10] H. Hofer, E. Zehnder, Symplectic Invariants and Hamiltonian Dynamics, Birkhäuser, Basel, 1994.

[11] S. Janeczko, A. Kowalczyk, On singularities in the degenerated symplectic geometry, Hokkaido Math. J. 19 (1990), 103-123.

[12] F. Lalonde, D. McDuff, The geometry of symplectic energy, Ann. of Math. (2) 141 (1995), 349-371.

[13] J. Martinet, Sur les singularités des formes différentielles, Ann. Inst. Fourier (Grenoble) 20 (1970), 95-178.

[14] D. McDuff, D. Salamon, Introduction to Symplectic Topology, Oxford Math. Monogr., The Clarendon Press, Oxford Univ. Press, New York, 1995. Second edition, 1998.

[15] D. McDuff, L. Traynor, The 4-dimensional symplectic camel and related results, in: Symplectic Geometry, D. Salamon (ed.), London Math. Soc. Lecture Notes Ser. 192, Cambridge Univ. Press, Cambridge, 1993, 169-182.

[16] J. Moser, On the volume elements on a manifold, Trans. Amer. Math. Soc. 120 (1965), 286-294.

[17] F. Pelletier, Singularités génériques d'une 2-forme et d'une 2-forme fermée sur une variété, C. R. Acad. Sci. Paris Sér. A-B 284 (1977), A1021-A1024.

[18] R. Roussarie, Modèles locaux de champs et de formes, Astérisque 30 (1975), 1-181.

[19] C. Viterbo, A proof of Weinstein's conjecture in $\mathbb{R}^{2 n}$, Ann. Inst. H. Poincaré Anal. Non Linéaire 4 (1987), 337-356.

[20] C. Viterbo, Symplectic topology as the geometry of generating functions, Math. Ann. 292 (1992), 685-710.

[21] M. Zhitomirskii, Typical Singularities of Differential 1-forms and Pfaffian Equations, Transl. Math. Monogr. 113, Amer. Math. Soc., Providence, 1992. 